\title{
Up Close: Max-Planck-Institute for Solid State Research in Stuttgart
}

\author{
E.E. Haller
}

\begin{abstract}
Editor's Note: This is the seventh article in a series focusing on the research capabilities and goals of interdisciplinary laboratories pursuing materials research in universities, industry, and government.
\end{abstract}

With the bestowal of the 1985 Nobel Prize upon Professor K. von Klitzing, one of the directors of the Max-Planck-Institute (MPI) for Solid State Research, there could no longer be any doubt - the Institute had made the ranks of prominent, top solid-state research centers in the world. Though Prof. K. von Klitzing was not a scientific member of the institute during his Nobel-prize-winning discovery of the Quantum Hall effect (Germans prefer to call it the von Klitzing effect), he nevertheless used the high magnetic fields and low temperatures available at the subsidiary of the MPI in Grenoble, France. He later joined the Stuttgart directors group, which now has 11 members.

What, then, is this MPI all about? I had the privilege of spending somewhat over half a year at this Institute doing research with two groups, free from all the bureaucratic burdens of home. During this time I had a chance to observe some of the inner workings of this scientific research establishment and also to participate actively in research. I became very impressed with the quality, breadth, and depth of the science and technology done there. Before beginning with a survey of the science, I want to summarize some history and give some statistical data.

The Max Planck Society was founded in 1948 , essentially following the course of the former Emperor Wilhelm Society, a society furthering science in Germany. Today, over $90 \%$ of the Society's income, around one billion DM in 1985, comes from government sources. The Society operates about 60 institutes with over 4,000 employees doing medical, physical, chemical, astronomical, and materials research, as well as institutes for history, education, psychiatry and law - a broad spectrum indeed!

The two MPIs in Stuttgart, housed in the same building which was completed in 1976, are focusing on solid-state research of nonmetallic materials and on metals research. Together they form the largest unit in the MPI system. They employ approximately 600 people, half of whom are scientists. Also, a significant number of graduate students perform their research at the two MPIs. On average, close to 100 guest scientists work at the two institutes at any given time. With which kind of U.S. laboratories can the MPIs in Stuttgart be compared? There is no exact U.S. analogue, though unclassified solid-state research performed by teams consisting predominently of professionals and supported by government funds can be found at many national laboratories. A strong graduate student component exists at several national laboratories as well. The considerable difference in size and support forces the MPIs to focus on a smaller number of research projects. On the other hand, the smaller size gives greater flexibility to adapt to new research trends and may keep the MPIs "young" for a long time.

The founding directors of the MPI for Solid State Research had a very clear goal in mind: to build a world class institute for advanced materials research which eventually would put German solid-state science back on the map after the devastating pre-World War II and World War II era. A famous and traditional MPI for metals research already existed, so it was decided that nonmetals were to be the research core of the new MPI. Today there are three solid-state theory groups, seven experimental groups, and a group running the famous high field magnet laboratory in Grenoble, France. The seven experimental groups can be divided into four groups pursuing solid-state physics and three groups working on solid-state chemistry. An additional nine "service groups," many doing their own research and development, prepare materials and provide characterization services. The excellent support, traditional for continental European research establishments, gives the researchers access to shops and to outstanding technological expertise.

The support is outstanding but also very organized and structured so that it removes some of the incentive for graduate students to try preliminary experiments on their own. In my opinion, they are not sufficiently encouraged to learn about instrumentation, electronics, and materials preparation "hands on." Together with tough electrical and building codes, it becomes difficult to try things out with a "jerry-rigged" setup and to find out quickly if the idea works - one aspect of laboratory work which gives great pleasure and satisfaction. One simply has to plan ahead and be well organized. Keeping this in mind, there is virtually nothing which cannot be done if one can just convince one of the directors of the merit and feasibility of an experiment.

In the following paragraphs, I will summarize the work done by the various groups. A brief article obviously cannot do full justice to all the aspects of a broad research effort. If more emphasis is given to one area than to another, it is solely because of personal preference and interest, not because of the importance or quality of the research.

\section{Theory}

The theory groups are headed by Professors $\mathrm{O}$. Andersen, the late $\mathrm{H}$. Bilz, and $P$. Fulde. Andersen's group works on the electronic structure of solids. Densityfunctional calculations are used to obtain information on structural and metal-insulator phase transitions. Excellent progress in the theoretical understanding of deep levels in silicon has been made recently by F. Beeler.

The group of the late $\mathrm{H}$. Bilz is studying the dynamical properties of solids which can be derived from their structure. The stability of semiconductor and transition metal surfaces are currently being investigated. Work on the physics of two-dimensional doped superlattices has been stimulated by work done in the experimental groups. Other areas of interest include ferroelectric systems and lattice excitations in amorphous semiconductors. Fulde's group concentrates on strongly and weakly electron-correlated systems. Heavy fermions are of special interest. Work on superconductivity, photoelectron spectroscopy, and electron-phonon interactions is pursued as well.

\section{Experimental Physics}

The experimental physics groups of $\mathrm{M}$. Cardona, L. Genzel, K. von Klitzing, and H.J. Queisser are strongly oriented toward semiconductor research. Major emphasis lies on multilayer systems, which are grown in-house by liquid phase epitaxy (LPE) or molecular beam epitaxy (MBE).

Continued 
The broad range of investigations includes thermoelectric and transport measurements at low temperatures and high magnetic fields; studies of the effects of dopants, defects, dislocations, and interfaces on the electronic, vibronic, and transport properties of silicon and III-V compound semiconductors; and far infrared studies of vibrational modes of biologically interesting molecules.

\section{Crystal Growth}

Special mention should be made regarding the excellent crystal growth facilities at the MPI. One of the world's leaders in LPE, E. Bauser, has developed a deep understanding of LPE-related processes and has produced the world's purest III-V semiconductor layers. A novel LPE centrifuge system, which is free of any sliders, transports the solvent liquids (maximum of four differently doped solvents) from one substrate to the next (maximum of four) by changes in angular velocity. This system has produced silicon $n-i-p-i$ structures with well over 100 layers which exhibit increasing crystalline perfection. One of the internationally renowned experts in MBE is K. Ploog. Together the two groups supply a large fraction of research samples used at this MPI. This special source of epitaxial layers is, of course, tapped by researchers all over the world and has led to numerous collaborations with universities and industrial laboratories. The leaders of the MPI have been stressing this service aspect from the very beginning, and from my observations, this role is taken very seriously.

\section{Solid-State Chemistry}

The solid-state chemistry groups of Professors A. Rabenau, H. von Schnering, and $\mathrm{A}$. Simon focus on chemical transport behavior and thermodynamic properties of solids which are investigated with solid-state electrochemistry or, as mentioned earlier, by MBE. Cluster compounds with polyanions and polycations are studied. Metal rich compounds are investigated in order to develop an understanding of structural and binding concepts and to foster the discovery of new groups of materials such as alkaline-metal suboxides, reduced rare earth halides, and transition metals cluster compounds.

This brief summary of the scientific programs illustrates the broad range of research interests reflected in the composition of the research teams. In order to encourage and foster interaction among the many scientists, engineers, and technologists, there are many devices for unplanned and often fruitful meetings and encounters. A large central stairway with small tea kitchens on every floor attracts a lot of traffic, especially at the beginning and ending of a work day and also during lunch breaks. Once per day, one can be sure to get hold of even the busiest person on the top floor, during the after-lunch coffee "klatsch." And a very practical, al- most necessary, commodity in view of the relatively remote location is the famous guest wing, where short stays of up to several days can be arranged.

I hope I have convinced you that the MPI in Stuttgart is a most lively place well worth considering for a visit during your next trip to Europe. And if you are interested in more than science, nearby Stuttgart offers much in regard to music, arts, museums, and Swabian hospitality.

\section{References}

1. Annual Report, October 1, 1984 - September 30, 1985, MPI for Solid State Research, Heisenbergstr. 1, 7000 Stuttgart 80, FRG.

2. Information booklet (Kurzinformation) in German, edited by E. Gmelin (MPI for Solid State Research) and E. Mann (MPI for Metals Research).

3. H.J. Queisser "Solids at Stuttgart," Mat. Res. Bull. 13 (1978) p. 369.

\section{E.E. Haller}

Lawrence Berkeley Laboratory and

University of California

Berkeley, CA 94720

Max-Planck-Institute for Solid State Research

Heisenbergstrasse 1

7000 Stuttgart 80

FRG

\section{NATO Advanced Study Institute Science and Technology of Fast Ion Conductors International School of Materials Science and Technology

\author{
July $1-15,1987$ \\ Erice, Italy
}

Two weeks of tutorials presented by a team of international experts will provide attendees with the background and perspective necesary to understand the novel, rapidly developing field of solid-state ionics and to conduct their own research.

The course is addressed to researchers and scientists from academia and industry in the fields of materials science, solid-state chemistry, electrochemistry, and physics with interests in sold-state ionics. Graduate students and scientists from allied fields in industry and government are also encouraged to participate. A limited number of scholarships may be available.

Deadline for Applications: March 15, 1987

Contact: Prof. Harry L. Tuller

Codirector, NATO Advanced Study Institute

Massachusetts Institute of Technology, Room 13-3126

77 Massachusetts Avenue

Cambridge, MA 02139

(617) 253-6890 